\title{
Autonomous Vision Based Detection of Non-stellar Objects Flying in Formation with Camera Point of View
}

\author{
Benn, Mathias; Jørgensen, John Leif
}

Published in:

Proceedings of 5th International Conference on Spacecraft Formation Flying Missions and Technologies

Publication date:

2013

Document Version

Publisher's PDF, also known as Version of record

Link back to DTU Orbit

Citation (APA):

Benn, M., \& Jørgensen, J. L. (2013). Autonomous Vision Based Detection of Non-stellar Objects Flying in Formation with Camera Point of View. In Proceedings of 5th International Conference on Spacecraft Formation Flying Missions and Technologies http://www.sffmt2013.org/PPAbstract/4152p.pdf

\section{General rights}

Copyright and moral rights for the publications made accessible in the public portal are retained by the authors and/or other copyright owners and it is a condition of accessing publications that users recognise and abide by the legal requirements associated with these rights.

- Users may download and print one copy of any publication from the public portal for the purpose of private study or research.

- You may not further distribute the material or use it for any profit-making activity or commercial gain

- You may freely distribute the URL identifying the publication in the public portal 


\title{
Autonomous Vision Based Detection of Non-stellar Objects Flying in Formation with Camera Point of View
}

\author{
As.Prof. M. Benn ${ }^{(1)}$, Prof. J. L. Jørgensen ${ }^{(2)}$ \\ ${ }^{(1)(2)}$ DTU Space, Elektrovej 327, 45253438, mb@space.dtu.dk, jlj@space.dtu.dk
}

Keywords: ASC, Advanced Stellar Compass, VBS, Vision Based Sensor, PRISMA

\begin{abstract}
The $\mu$-Advanced Stellar Compass ( $\mu \mathrm{ASC}$ ) is a camera system which autonomously can provide attitude information based on the stars. An extended functionality of the $\mu A S C$, called the Vision Based Sensor (VBS), enables the camera system to render rendezvous and docking navigation information for a formation flying spacecraft.

The VBS system facilitates different operation modes, enabling 6DoF solutions for spacecraft flying in close proximity, and inertial Line-of-Sight (LoS) solutions for any spacecraft reflecting more light than an Mv7 star.

This paper will focus on the LoS solutions of the VBS system, with a thorough analysis of inflight performance and future mission aspects.
\end{abstract}

The VBS system is currently running on the PRISMA mission launched in 2010, where it functions as a part of the instrument package forming the rendezvous and docking navigation test bench for the Main spacecraft. Here the VBS system has been enabled based on the scheduled flight maneuvers in between the two spacecraft, autonomously providing the Main spacecraft with pose and position or LoS information of the Target spacecraft.

During different phases of the mission, full centroid information was captured from the Camera Head Units (CHUs) of the $\mu$ ASC, which for these periods have enabled on-ground VBS reprocessing and deeper investigation of the detected objects and their statistical occurrences.

The PRISMA mission facilitates a total of four CHUs where two are dedicated for normal startracker operation and the other two CHUs are dedicated for VBS operation, one for short range constellation and one for far range inertial LoS operation.

The captured data used for the analysis presented in this paper, includes centroids from the VBS CHU dedicated for inertial LoS operation and from the two standard startracker CHUs. By combining the data from these three $\mathrm{CHUs}$, a total of 300 hours of operation distributed over the entire year is included in the thorough data analysis and performance characterization.

The analysis has provided a great overview of performance for the LoS detection capabilities of the VBS system. This includes inertial pointing accuracy vs range for a known target spacecraft, robustness of the autonomous behavior and frequency of detected non-stellar objects flying in low-earth orbit.

This paper will present the methods of analysis used for treading the vast amount of inflight data together with the outcome of the VBS algorithms and the performance of the system. In conclusion the paper provides expected performance overview for use 
of the VBS instrument for future low-earth orbit missions as well as for deep space missions, including sample return.

\section{Vision Based Sensor}

The Vision Based Sensor (VBS) is an instrument capable of autonomously determining relative pose and position between two spacecrafts flying in close formation, or provide line-of-sight solutions for distant flying objects/spacecrafts. Solution information is based on digital images captured using cameras placed on a spacecraft tracking Non-stellar Objects (NSOs) of interest in the Field-of-View (FOV) of the imaging camera.

The VBS system is realized as an extension module to an already space proven instrument, the $\mu$-Advanced Stellar Compass ( $\mu A S C$ ), which is capable of fully autonomous delivery of high precision attitude references based upon star observations [2]. The VBS system utilizes the $\mu A S C$ imaging platform either with specific VBS Camera Head Units (CHUs) for Short Range (SR) operations or using the standard star tracker CHUs for Far Range (FR) operations.

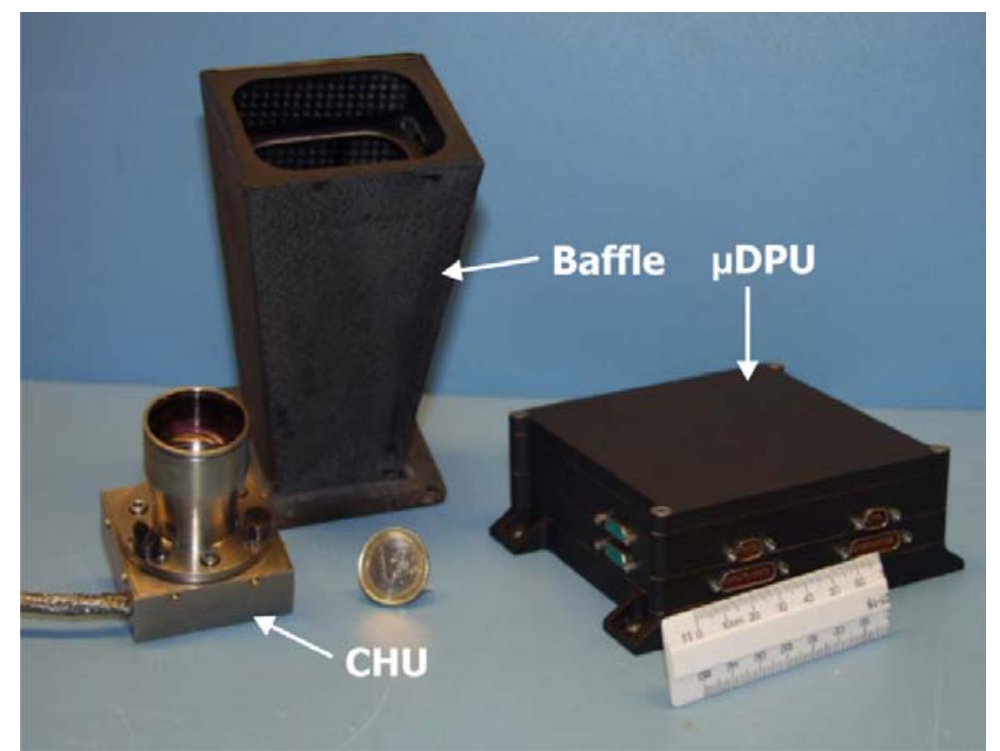

Figure 1: System elements of the $\mu$-Advanced Stellar Compass, depicting a CHU, a baffle and a redundant double $\mu D P U$.

By providing either pose and position of a spacecraft or line-of-sight information of NSOs to the onboard Guidance, Navigation and Control (GNC) unit, the VBS system enables closed loop formation flying, rendezvous and docking capabilities.

The PRISMA satellite mission is the first to utilize the VBS system on the implemented $\mu$ ASC of the Main spacecraft, using the VBS solutions in the GNC core to perform closed loop formation flying with the Target spacecraft. The PRISMA project, which is led by the Swedish Space Cooperation [1], is a technical demonstration mission, constituting of an in-orbit test bed for GNC algorithms as well as sensors for rendezvous and closed-loop formation flying based on a Main and a Target spacecraft. The VBS system is one of three systems on the PRISMA mission for relative position determination of the Target spacecraft. 
The configuration of the $\mu$ ASC for PRISMA consists of a total of four CHUs:

- One standard low light CHU capable of detecting stars and is used by the VBS system for far range operations.

- One high light CHU with a fixed aperture and filter included and is used by the VBS system for close range operations.

- And two standard star tracker CHUs reserved for attitude determination.

The placement of the VBS CHUs on the PRISMA spacecraft is illustrated in Figure 2, where the two standard CHUs are placed on the backside of the Main spacecraft.

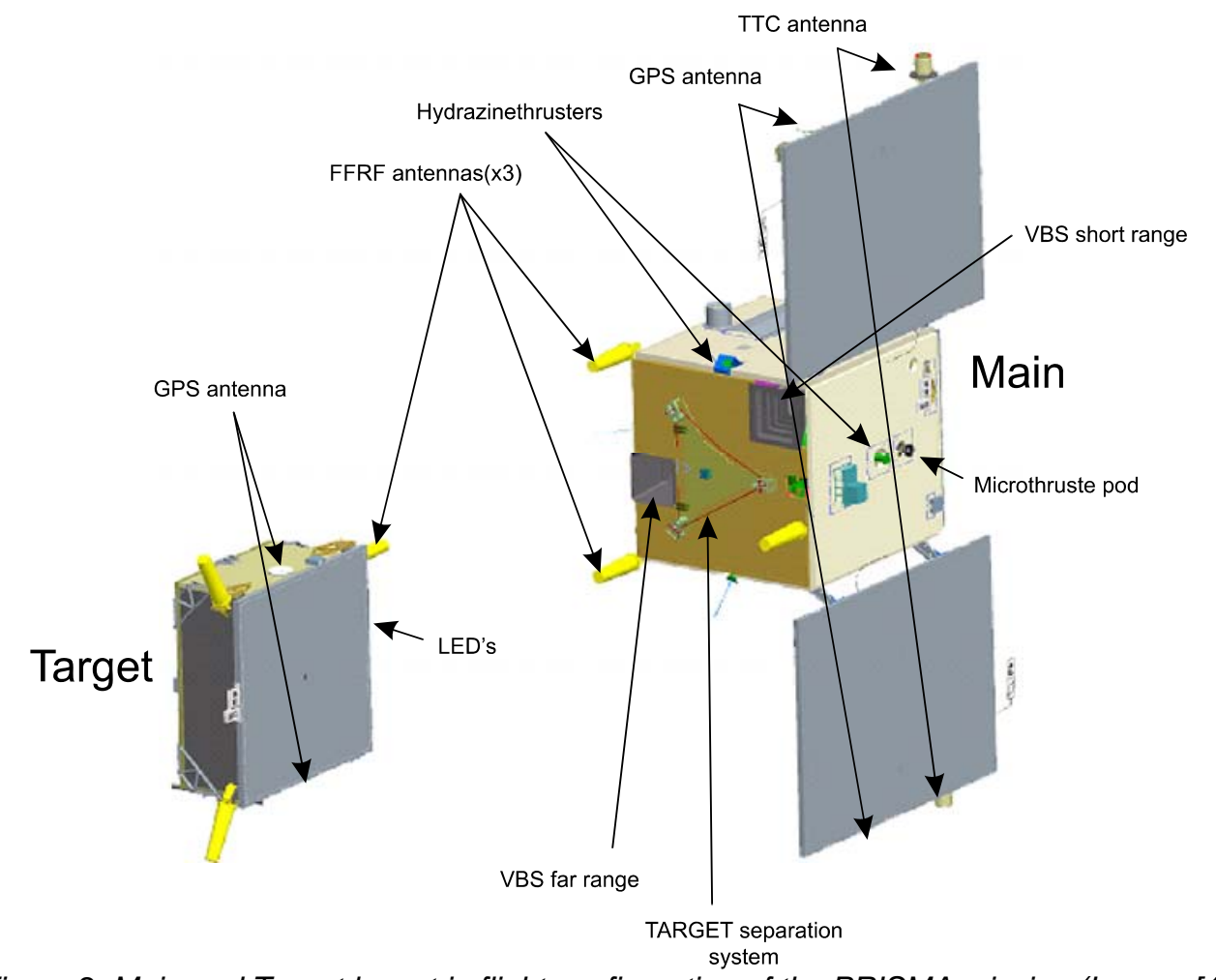

Figure 2: Main and Target layout in flight configuration of the PRISMA mission (Image: [1])

Further information regarding the VBS system on PRISMA is presented in $[3,4,5,6]$.

\section{Rerun of VBS Algorithms on Recorded Centroids}

The VBS system as implemented on PRISMA is designed to output only the best detected Target spacecraft candidate for line-of-sight solutions. The VBS does however internally keep track of several object trajectories simultaneously, which are of interest for analyzing the availability of tracked objects, frequency of detections and have precise information of the best candidate for a Target object.

An on-ground VBS rerun of the inflight recorded centroids from the PRISMA mission has provided such information, which are the data presented in this paper.

Three of the CHUs on PRISMA are capable of determine attitudes and provide centroid information of the detected objects on the image sensor, namely, CHU A, B 
and $\mathrm{C}$. The centroid information is available from the $\mu \mathrm{ASC}$ on request, and on the PRISMA mission a total of $\sim 300$ hours of centroids have been recorded at $2 \mathrm{~Hz}$.

By combining these centroids with the determined attitude information, the centroids can be split up into detected stars and into detected Non-stellar Objects. Stars are already known to the VBS system whereas the Non-stellar Objects are the objects of interest for tracking.

In Figure 3 is illustrated the time periods for which the $\mu A S C$ has detected NSOs during the PRISMA mission. The splitting into the different $\mathrm{CHUs}$ are illustrated due to the fact that the CHUs have had different purposes during the PRISMA mission.

Likewise, in Table 1 is given the total number of NSOs for reprocessing by the VBS algorithms.

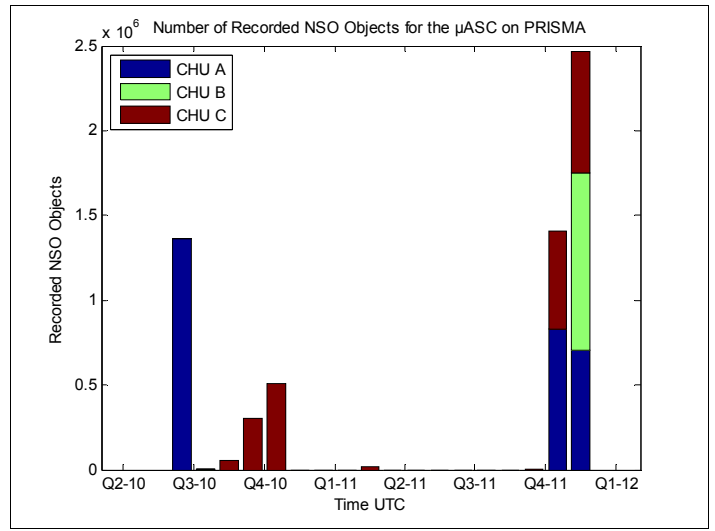

\begin{tabular}{|c|c|}
\hline CHU A & 2898486 \\
\hline CHU B & 1050754 \\
\hline CHU C & 2177309 \\
\hline Total & $\mathbf{6 1 2 6 5 4 9}$ \\
\hline
\end{tabular}

Table 1: Total number of Non-stellar Objects detected.

Figure 3: Recorded Non-stellar Objects from PRISMA mission.

\section{Tracked Objects}

The VBS algorithms used for rerunning the NSOs are similar to the inflight algorithms on the $\mu \mathrm{ASC}$ with the addition of included probes for extracting the state of tracked objects.

In order to determine a correlation between the detected NSOs, the VBS are accepting objects that are moving within a certain bandgap of inertial angular velocities. As soon as an NSO has 3 sequential detections, the NSO is marked as an object for tracking. This gives that the greater the tracking history, the greater the confidence of the tracked object is.

The inertial velocity bandgap is based on the fact that the PRISMA satellites are

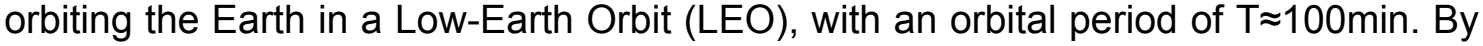
assuming that the Main spacecraft are following the Target spacecraft in a circular orbit, the inertial angular velocity of Target as observed by Main can be expressed by:

$$
\omega=\frac{\delta \theta}{\delta T}=\frac{360^{\circ}}{100 \min }=216 \frac{n}{s}
$$

In order to detect as many objects as possible for the rerun, the bandgap limits are set in the range of 100 "/s to 5000 "/s for input to the VBS algorithms. 


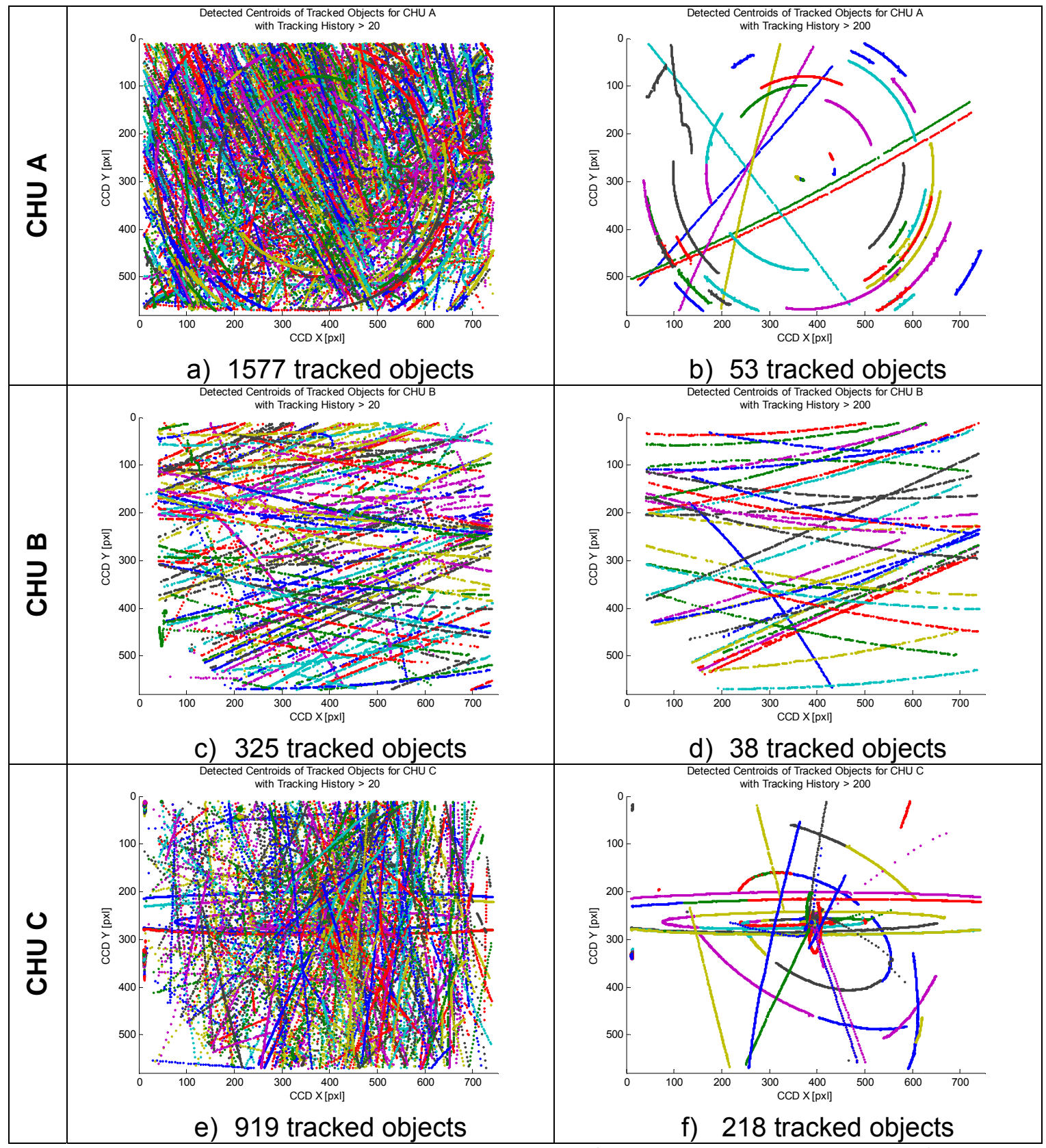

Figure 4: Detected centroids of objects tracked by the VBS system. The data is divided on CHUs. Left column for tracking history $>20$ and right column for tracking history $>200$. The colors separate the different trackings.

In Figure 4 are illustrated all the detected tracks on the CCD plane as seen by the three $\mathrm{CHUs}$. The difference in the tracks inbetween the CHUs are clearly given, which are due to the different usage of the CHUs:

- $\mathrm{CHU}$ A has been recording centroids during an inertial pointing stability test pointing perpendicular to the orbit plane. This has resulted in tracking of relative circular motions of objects in almost parallel orbits as PRISMA.

- $\mathrm{CHU} \mathrm{B}$ has been recording centroids during normal operation while pointing $\sim 45^{\circ}$ off the orbit plane. 
- $\mathrm{CHU} \mathrm{C}$ has been recording centroids mainly with the Target spacecraft in FoV while pointing along the orbit flight path.

In Figure 5 are illustrated the tracking characteristics with respect to tracking history and angular velocities for the three CHUs. The long durations of steady inertial pointings with $\mathrm{CHU}$ A have resulted in accomplishing great tracking history of detected objects. Additionally, fast moving objects following along-track the spacecraft are detected. CHU B reflect tracking of objects that are similar to each other, while $\mathrm{CHU} \mathrm{C}$ is very likely to have the Target spacecraft available for tracking, illustrated by the peak in angular velocities.

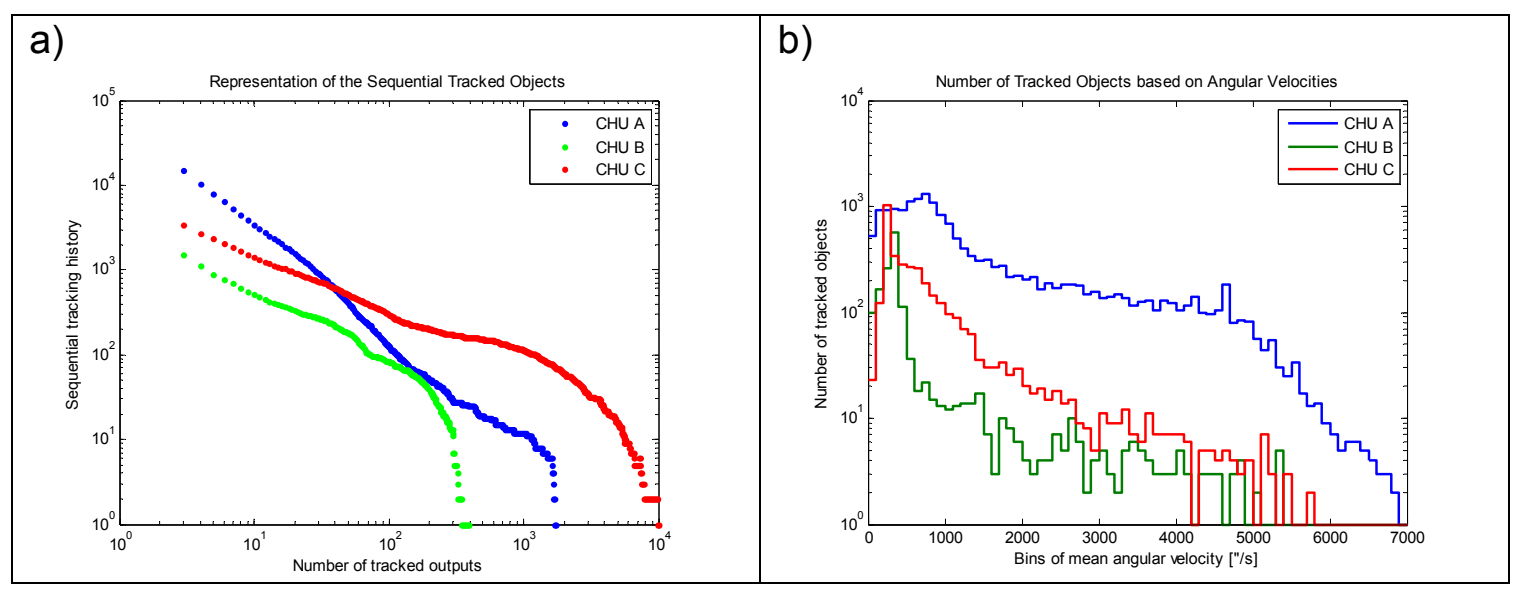

Figure 5: Tracking characteristics for the three PRISMA CHUs. (a)Tracking history, (b) Angular velocity.

\section{Frequency of Tracked Objects}

While orbiting the Earth at a rather constant rate, it is desired to analyze at which frequency newly detected objects occur. This will provide with information about how many objects will get into detection range and how often they occur.

In Figure 6 are illustrated the time difference between newly tracked objects with three different limitations, namely, for tracking histories of 10, 100 and 1000.

For objects with a tracking history of 10 is not yet in a stable history range for providing great confidence on the track. It is seen that these occurs at frequency peaks around $0.5 \mathrm{~s}, 1 \mathrm{~s}, 2 \mathrm{~s}$ and $4 \mathrm{~s}$, which are closely related to the updating frequency of $2 \mathrm{~Hz}$ for the $\mu \mathrm{ASC}$ imaging system.

For the case with a minimum of 100 in history tracking, the peak shifts since the newly detected trackings with this history occurs less frequently. Furthermore, a peak at the frequency of the orbital period of PRISMA occurs, which is due to repeated detections of objects flying in similar or crossing orbits.

The last illustration including objects with a tracking history greater than 1000 , shows that the peak shifts even closer to the orbital period of PRISMA. The main peak is still below the orbital period mark, which gives that newly tracked objects with high confidence are occurring several times over just one orbit period. 


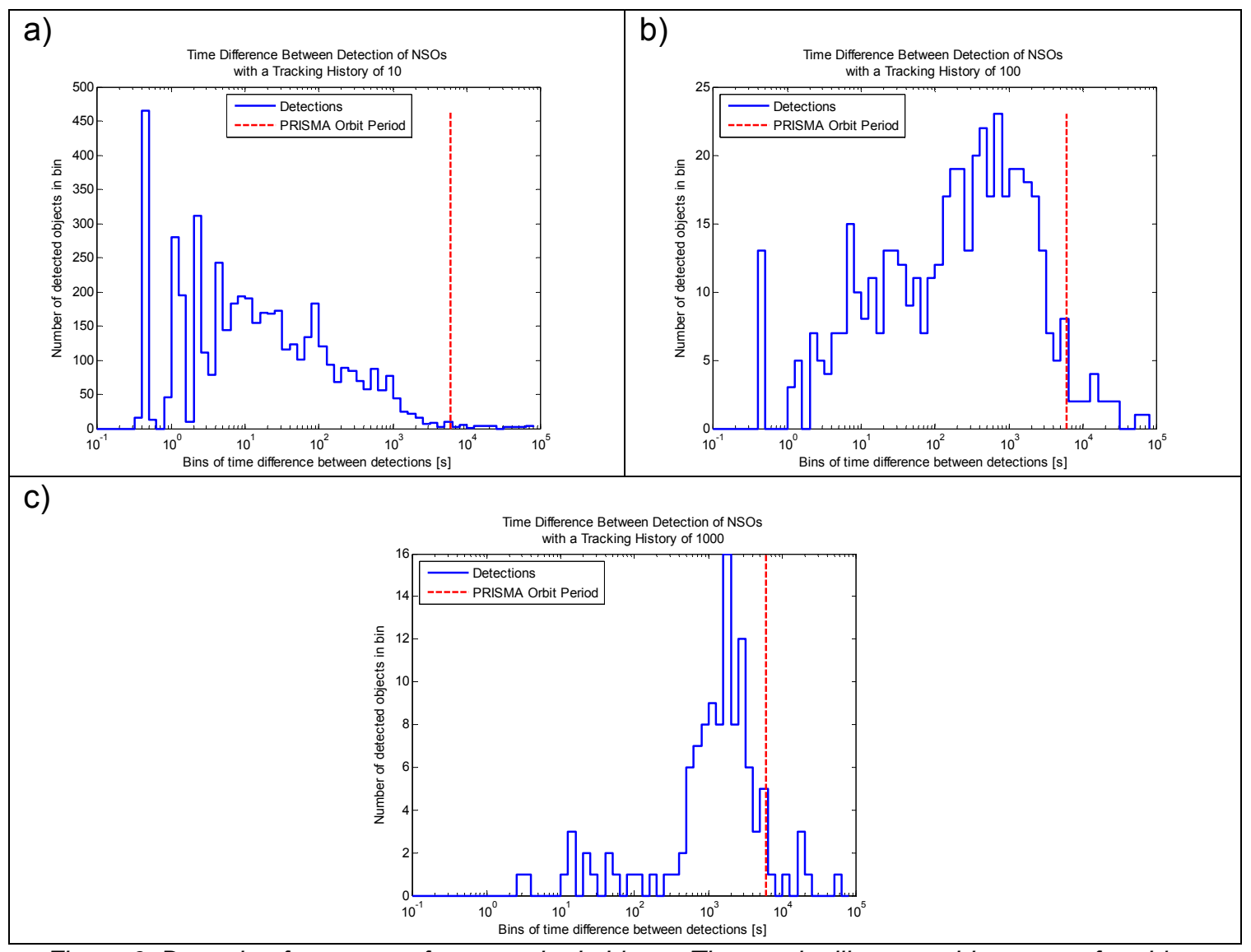

Figure 6: Detection frequency of new tracked objects. The graphs illustrates histograms for objects with tracking history of (a) 10 and above, (b) 100 and above, (c) 1000 and above.

\section{Pointing Accuracy towards Known Target}

In order to analyze performance of pointing accuracy, data including tracking information of the Target spacecraft is extracted from $\mathrm{CHU} \mathrm{C}$ in order to have a known Target candidate. The data extracted are illustrated in Figure 7 for all the different trackings.

a)

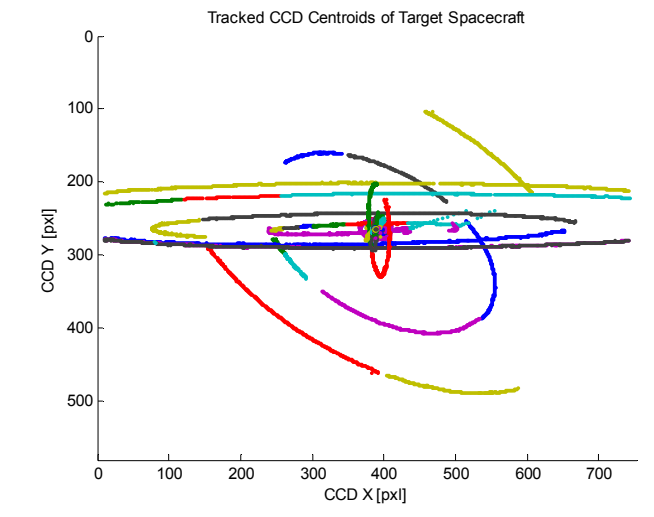

b)

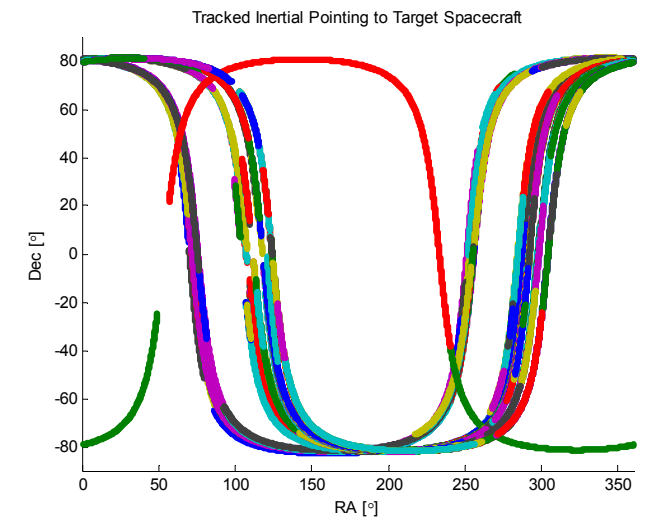

Figure 7: Tracking of known Target spacecraft. (a) The tracks at detected by the CCD. (b) The inertial pointing of the tracking. The colors separate the different trackings. 
The VBS algorithms are performing running average of the tracked objects in order to predict the next possible inertial pointing towards the object. In Figure 8 is illustrated the comparison between the VBS predicted inertial pointing and the actual detected inertial pointing towards the tracked object, together with the expected maximum deviation over distance.

The theoretical expected maximum is based on the angular size of the Target spacecraft from where reflections can occur, together with the centroiding accuracy of the $\mu \mathrm{ASC}$ system. It is seen that for distances below $10 \mathrm{~km}$ the deviation is within the expected maximum, but the few samples at $\sim 30 \mathrm{~km}$ is just on the boundary. This can be due to acceleration of the Target spacecraft during approach and recede maneuvers.

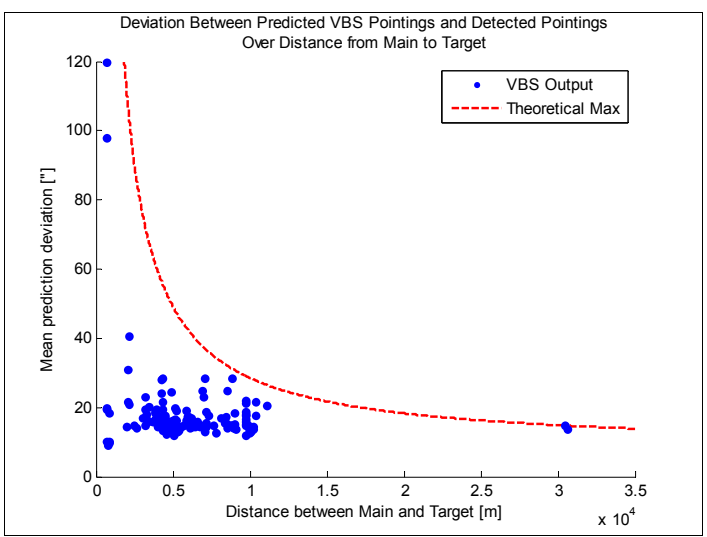

Figure 8: Detection precision vs. the distance between Main and Target.

\section{Future Implementations}

The VBS algorithms are currently flying on the JUNO mission on-route to Jupiter, which will start orbiting Jupiter in 2016. The main objectives for the VBS system are to detect asteroids on its route to Jupiter, as well as detection of minor moons when arriving at Jupiter.

Next generation of the VBS system is planned for Proba-3 flying in a Highly Elliptical Orbit (HEO) with a configuration for LoS solutions and 6DoF solutions for the VBS system, providing an Occulter spacecraft with GNC information for flying in controlled formation with a Coronagraph spacecraft. This mission has to operate at a distance of $150 \mathrm{~m}$, which is three times the maximum distance experienced for 6DoF solutions on the PRISMA mission. This requires a redesign and optimization of the imaging $\mathrm{CHU}$ for the given scenario.

Other Rendezvous and Docking missions are already on the drawing board for including VBS functionalities, using $\mathrm{CHUs}$ which includes a $\mu$-Inertial Reference Unit (MIRU) that provides high frequency attitude solutions. This will accelerate both inertial attitude determination and expand the 6DoF solution range for the VBS system. 


\section{Conclusion}

By post-processing of inflight recorded centroid data, it has been shown that the VBS system is very much capable of detecting and tracking several Non-stellar Objects simultaneously.

From a satellite orbiting the Earth, all satellites detectable by the star tracker CHUs can be tracked as soon as their inertial angular movement, with respect to the Main satellite, fits within the bandpass values provided to the VBS system.

The VBS system is capable of providing high accuracy inertial pointings of all tracked objects in combination with key tracking information, that can be used for determine the confidence and precision of the detected object.

Furthermore, dependent on which NSO object that are of interest to search for, different boresight pointings relative to flight path have shown a wide covering range for tracking of fast and slow moving NSOs.

By providing inertial pointing towards any Non-stellar Object to a GNC core, approach maneuvers can easily be performed in the right direction. The VBS system is fully autonomous and can detect objects as soon as they can be detected by the star tracker CHUs. This is especially useful for missions with limited commandability, such as e.g. Sample-Return missions.

\section{References}

1. Persson, S., Bodin, P., Gill, E., Harr, J. and Jørgensen, J.L. PRISMA - An Autonomous Formation Flying Mission, European Space Agency, 625 SP, 2006.

2. Denver, T., Jørgensen, J.L., Michelsen, R. and Jørgensen, P.S. MicroASC Star Tracker Generic Developments, Small Satellite Systems and Services Symposium, Chia Laguna, Sardinia, Italy, 25-29 Sept. 2006.

3. Benn, M. Design of a Visual Rendezvous and Docking Navigation Sensor, Master Thesis Project at the Danish Technical University, 2007.

4. Benn, M. Short Range Pose and Position Determination of Spacecraft Using a $\mu$ Advanced Stellar Compass. $3^{\text {rd }}$ International Symposium on Formation Flying, Missions and Technologies, ESTEC, Apr 2008

5. Benn, M. Range Management of a Vision Based Rendezvous and Docking Navigation Sensor. International Astronautical Congress, Daejeon, Korea, Oct 2009.

6. Bjarnø, J. B. Short range camera for Rendezvous in Space, Performance and scenario study of the VBS system, DTU Space, Dec 2005. 